\title{
OELJEKLAUS-TOMA MANIFOLDS ADMITTING NO COMPLEX SUBVARIETIES
}

\author{
Liviu Ornea And Misha Verbitsky
}

To Professor Vasile Brînzănescu at his sixty-fifth birthday

\begin{abstract}
The Oeljeklaus-Toma (OT)-manifolds are complex manifolds constructed by Oeljeklaus and Toma from certain number fields, and generalizing the Inoue surfaces $S_{m}$. On each OT-manifold we construct a holomorphic line bundle with semipositive curvature form $\omega_{0}$ and trivial Chern class. Using this form, we prove that the OTmanifolds admitting a locally conformally Kähler structure have no non-trivial complex subvarieties. The proof is based on the Strong Approximation theorem for number fields, which implies that any leaf of the null-foliation of $\omega_{0}$ is Zariski dense.
\end{abstract}

\section{Contents}

1. Introduction 1

1.1. OT-manifolds and their subvarieties 1

1.2. Number theory and the construction of OT-manifolds 3

2. The weight bundle of an OT-manifold 4

3. Complex subvarieties in LCK OT-manifold 6

$\begin{array}{ll}\text { Acknowledgments } & 8\end{array}$

$\begin{array}{ll}\text { References } & 8\end{array}$

\section{Introduction}

1.1. OT-manifolds and their subvarieties. The Oeljeklaus-Toma (OT)manifolds are an important class of compact complex manifolds not admitting a Kähler metric. They were discovered by Oeljeklaus and Toma in 2005 [5]. The construction of OT-manifolds uses the Dirichlet unit theorem from number theory (Subsection 1.2; see [7] for additional details of this construction and many related questions). Starting from a degree 3 number field, one obtains a two-dimensional OT-manifold known as Inoue surface $S_{m}$ (see [2]).

For some number fields, the OT-manifolds are locally conformally Kähler (LCK). An LCK structure on a complex manifold is a Kähler metric on its universal cover $\tilde{M}$, such that the deck transform maps act on $\tilde{M}$ by homotheties. The OT-manifolds serve an important function in the theory of LCK manifolds, providing a counterexample

Received by the editors November 26, 2010.

2000 Mathematics Subject Classification. 53C55.

Key words and phrases. Locally conformally Kähler manifold, Kähler potential, positive bundle, complex subvariety, Inoue surface. 
to a longstanding conjecture of I. Vaisman, [9], who asked whether there exists a compact, non-Kähler LCK-manifold $M$ with all odd Betti numbers even: $b_{2 p+1}(M) \equiv$ $0 \bmod 2$. The OT manifolds in dimension 3 are the only known examples of compact LCK-manifolds with even odd Betti numbers, $b_{1}=b_{5}=2, b_{3}=0$.

An OT-manifold is LCK if it is constructed from a number field $K$ which has precisely two complex (non-real) embeddings, that is, two distinct homomorphisms $K \stackrel{\sigma, \bar{\sigma}}{\longrightarrow} \mathbb{C}$. If the OT manifold has at least four complex embeddings and exactly one real, then it is not LCK. The remaining case is not yet decided.

Inoue surfaces $S_{m}$ have no curves. We give a generalization of this theorem, proving that an OT-manifold that is LCK has no non-trivial complex subvarieties. In particular, it has no non-constant meromorphic functions (as a meromorphic function without polar set would be holomorphic, and hence constant).

Question 1.1. Is there any OT (non-LCK) manifold that has non-constant meromorphic functions?

The idea of the proof of this result is quite simple. We construct a holomorphic Hermitian line bundle, called the weight bundle, on any OT-manifold $M$. This bundle is topologically trivial, and has semipositive curvature form $\omega_{0}$. The weight bundle also admits a flat connection, compatible with the holomorphic structure.

To learn about complex subvarieties of an OT-manifold, we study the zero-foliation $\Sigma$ of $\omega_{0}$, proving that all its leaves are Zariski dense in $M$. For an OT-manifold $M$ constructed from a number field $K$ admitting exactly $2 t$ distinct complex (non-real) embeddings to $\mathbb{C}$, the leaves of $\Sigma$ are $t$-dimensional. When $t=1, M$ is LCK, and $\Sigma$ is one-dimensional. In this case, we prove that for any positive-dimensional complex subvariety $Z \subset M, Z$ contains with each point $z \in Z$ the leaf $\Sigma_{z}$ passing through $z$. Since all leaves of $\Sigma$ are Zariski dense, the same is true for $Z$.

The weight bundle $L$ is quite useful for many other purposes. As it was done in [12], one can take the $\alpha$-th tensor power of $L$, denoted by $L^{\alpha}$, for any real $\alpha$; this power is well defined, because $L$ is equipped with a natural $C^{\infty}$-trivialization. The Gauduchon degree $\operatorname{deg}_{\mathrm{g}}$ of $L^{\alpha}$, taken with respect to any Gauduchon metric, satisfies $\frac{1}{\alpha} \operatorname{deg}_{\mathrm{g}} L^{\alpha}=\operatorname{deg}_{\mathrm{g}} L>0$, hence $M$ admits a line bundle with any prescribed Gauduchon degree. This implies, in particular, that the connected component of the Picard group $\operatorname{Pic}(M)$ is non-compact. Also, this implies that any vector bundle on $M$ has degree zero after tensoring with an appropriate power of $L$; this is useful for the study of Hermitian-Einstein bundles on $M$, providing useful tools for the classification of stable bundles, and, eventually, coherent sheaves on $M$.

A similar argument was used in [12] to study holomorphic vector bundles and subvarieties on homogeneous elliptic fibrations, such as Calabi-Eckmann manifolds and quasi-regular Vaisman manifolds. We pose two questions, very much unsolved, but quite natural in the context presented by [12] and the present paper. Notice that from their construction it is clear that OT-manifolds are affine flat, that is, equipped with a flat, affine, torsion-free connection.

It is shown in [5, Remark 1.7] that some OT manifolds admit a holomorphic foliation with compact leaves which are again OT manifolds. Hence, it is natural to pose the following: 
Question 1.2. Are the ones described in [5, Remark 1.7] the only OT manifolds with compact complex subvarieties? Can we classify these subvarieties? Are they always totally geodesic with respect to the flat affine connection?

Question 1.3. Does there exist a stable holomorphic vector bundle of rank $>1$ on any OT-manifold of dimension $>2$ ? Do all holomorphic vector bundles admit a flat connection, compatible with the holomorphic structure?

Remark 1.1. It is well known that generic complex tori have no non-trivial complex subvarieties. In [11], it was shown that all stable bundles on a generic complex torus of dimension $>2$ have rank 1 , and all holomorphic vector bundles admit flat connections. As for compact complex surface of non-Kählerian type, it is proven in [13] that stable holomorphic two-bundles with $c_{1}=0$ and $c_{2}=n$ exist for any $n>0$.

1.2. Number theory and the construction of OT-manifolds. Let $[K: \mathbb{Q}]$ be a number field, that is, a finite extension of $\mathbb{Q}$, of degree $n$, with $\sigma_{1}, \ldots, \sigma_{s}$ the real embeddings of $K$ into $\mathbb{C}$, and $\sigma_{s+1}, \ldots, \sigma_{n}$ the complex embeddings. Since the complex embeddings of $K$ into $\mathbb{C}$ occur in pairs of complex conjugate embeddings, the number $n-s$ is even, $n-s=2 t$. Let $\sigma=\left(\sigma_{1}, \ldots, \sigma_{n}\right): K \rightarrow \mathbb{C}^{s+t}$ be the corresponding group homomorphism.

Let $\mathcal{O}_{K}$ be the ring of algebraic integers of $K, \mathcal{O}_{K}^{*}$ its multiplicative group of units and $\mathcal{O}_{K}^{*,+}$ the group of units which are positive in all the real embeddings of $K$.

Denote by $\mathbb{H}$ the upper complex half-plane. Using the Dirichlet's unit theorem, Oeljeklaus and Toma proved that $\mathcal{O}_{K} \rtimes \mathcal{O}_{K}^{*,+}$ acts freely on $\mathbb{H}^{s} \times \mathbb{C}^{t}$ by

$$
\begin{aligned}
& T_{a}\left(z_{i}\right)=\left(z_{i}+\sigma_{i}(a)\right), \quad i=1, \ldots, s+2 t, \quad a \in \mathcal{O}_{K}, \\
& R_{u}\left(z_{i}\right)=\left(\sigma_{i}(u) z_{i}\right), \quad i=1, \ldots, s+2 t, \quad u \in \mathcal{O}_{K}^{*,+} .
\end{aligned}
$$

(see $[5,7])$. Moreover, an admissible subgroup $U \subset \mathcal{O}_{K}^{*,+}$ can always be found such that the action of $\Gamma:=\mathcal{O}_{K} \rtimes U$ is also properly discontinuous. For $t=1$, every $U$ of finite index in $\mathcal{O}_{K}^{*,+}$ has this property.

Definition 1.1. The manifold $M_{K}:=\left(\mathbb{H}^{s} \times \mathbb{C}^{t}\right) / \Gamma$ is called an OT manifold. It is a compact complex manifold of dimension $s+2 t$.

For $s=t=1, M_{K}$ reduces to an Inoue surface $S_{m}$ (where $m$ is a matrix in $\operatorname{SL}(3, \mathbb{Z}))$, see $[2]$. The corresponding number field $K$ is $\mathbb{Q}[T] / P_{m}(t)$, where $P_{m}(t)$ is the characteristic polynomial of the matrix $m$. It is shown in [5] that the manifolds $M_{K}$ are never Kähler, but that for $t=1, M_{K}$ is an LCK manifold (see [1] and the more recent survey [6] for definitions and results in LCK geometry). We briefly explain the construction of this LCK metric.

Clearly, the function $\psi(z)=\prod_{i=1}^{s}\left(\mathrm{im} z_{i}\right)+\left|z_{s+1}\right|^{2}$ is plurisubharmonic on $\mathbb{H}^{s} \times \mathbb{C}$. It defines the Kähler form $\Omega:=\partial \bar{\partial} \psi$ on $\mathbb{H}^{s} \times \mathbb{C}$. The group $\Gamma$ acts on $\left(\mathbb{H}^{s} \times \mathbb{C}, \Omega\right)$ by homotheties:

$$
\begin{aligned}
& T_{a}^{*} \Omega=\Omega, \\
& R_{u}^{*} \Omega=\left|\sigma_{s+1}(u)\right|^{2} \Omega .
\end{aligned}
$$


Let now $\chi: \Gamma \rightarrow \mathbb{R}^{>0}$ be the character $\chi(\gamma)=\frac{\gamma^{*} \Omega}{\Omega}$. We call automorphic any $p$-form $\eta \in \Lambda^{p}\left(\mathbb{H}^{s} \times \mathbb{C}\right)$ which satisfies $\gamma^{*} \eta=\chi(\gamma) \eta$. For any automorphic function $\varphi$ on $\mathbb{H}^{s} \times \mathbb{C}$, the quotient $\frac{\Omega}{\varphi}$ is $\Gamma$-invariant and hence projects to an LCK metric $\omega$ on $M_{K}$. This form satisfies the equation $d \omega=\theta \wedge \omega$, for the closed 1-form $\theta$ (called the Lee form), which is the projection on $M_{K}$ of $\tilde{\theta}=-d \log \varphi$ :

$$
d \omega=-\frac{d \varphi}{\varphi^{2}} \wedge \tilde{\omega}=-d(\log \varphi) \wedge \omega .
$$

It is easily seen that the function $\varphi=\prod_{i=1}^{s}\left(\mathrm{im} z_{i}\right)^{-1}$ is automorphic, and hence it produces a LCK metric on $M_{K}$ as described above. This LCK metric generalizes the one constructed by Tricerri on $S_{m},[8]$.

The main result of this paper shows that, just as Inoue surfaces $S_{m}$ have no complex curves, OT-manifolds have no complex subvarieties:

Theorem 1.1. Let $[K: \mathbb{Q}]$ be a number field of degree $n=s+2$, with s real embeddings and two complex embeddings, and $M_{K}$ the corresponding LCK OT-manifold. Then $M_{K}$ has no non-trivial complex subvarieties.

Proof. See Theorem 3.1.

Corollary 1.1. The LCK OT-manifold $M_{K}$ has no non-constant meromorphic functions.

\section{The weight bundle of an OT-manifold}

Definition 2.1. Let $[K: \mathbb{Q}]$ be a number field of degree $n=s+2 t$, with $s$ real embeddings and $2 t$ complex embeddings, and $M_{K}=\mathbb{H}^{s} \times \mathbb{C}^{t} / \Gamma$ the associated OTmanifold. Denote by $z_{1}, \ldots, z_{s}$ the standard complex coordinates on $\mathbb{H}^{s}$, and let $\tilde{\theta}:=-d \log \prod_{i=1}^{s}\left(\operatorname{im} z_{i}\right)$. It is easy to see that the form $\tilde{\theta}$ is $\Gamma$-invariant. Therefore it is obtained as a lift of a form $\theta$, called the Lee form of the OT-manifold. When $t=1$, this is the Lee form constructed above.

Let $M_{K}$ be an OT-manifold, and $\theta$ its Lee form. Consider a trivial Hermitian line bundle $L$ with connection $\nabla:=\nabla_{0}+\sqrt{-1} \theta^{c}$, where $\theta^{c}:=I \theta$, and $\nabla_{0}$ is the trivial connection on $L$. Clearly, $\nabla$ is Hermitian, and $\nabla^{0,1}=\bar{\partial}+\theta^{0,1}$, where $\theta^{0,1}$ is the $(0,1)$-part of $\theta$.

Claim 2.1. In these assumptions, the curvature $\omega_{0}$ of $\nabla$ is $-\sqrt{-1} d \theta^{c}$. Moreover, this form is of type $(1,1)$.

Proof. A simple computation shows that in the standard coordinates $z_{1}, \ldots z_{s}$, $z_{s+1}, \ldots z_{s+t}, \omega_{0}$ can be written as follows:

$$
\omega_{0}=\sqrt{-1} \partial \bar{\partial} \log \varphi=\sqrt{-1} \sum_{i=1}^{s} \frac{d z_{i} \wedge d \bar{z}_{i}}{\left|\operatorname{im} z_{i}\right|^{2}}
$$


Definition 2.2. Let $M_{K}$ be an OT-manifold, and $L$ the holomorphic Hermitian bundle defined above. Then $L$ is called the weight bundle of $M_{K}$.

We restate Claim 2.1 as

Theorem 2.1. Let $M_{K}$ be an OT-manifold, and L its weight bundle with the holomorphic Hermitian structure and the Chern connection $\nabla$ defined above. Consider the form $\omega_{0}:=\sqrt{-1} \nabla^{2}$. Then $\omega_{0}$ is a semi-positive form, which can be written in the standard coordinates $z_{1}, \ldots z_{s}, z_{s+1}, \ldots, z_{s+t}$ as follows:

$$
\omega_{0}=\sqrt{-1} \partial \bar{\partial} \log \varphi=\sqrt{-1} \sum_{i=1}^{s} \frac{d z_{i} \wedge d \bar{z}_{i}}{\left|\operatorname{im} z_{i}\right|^{2}}
$$

Remark 2.1. The Vaisman manifolds are, by definition, LCK manifolds $(M, I, g)$ satisfying the additional condition $\nabla^{g} \theta=0$, where $\nabla^{g}$ is the Levi-Civita connection of an LCK metric $g$. For all Vaisman manifolds, the two-form $\omega_{0}=d \theta^{c}$ is semipositive, being zero only on the direction of $\theta^{\sharp}-I \theta^{\sharp}$. This is a general fact, proven in [10], independent of the particular form of $\theta$. OT-manifolds are far from being Vaisman (they never admit any Vaisman metric), but the particular expression of their Lee form gives $\omega_{0}$ the same property as for Vaisman manifold. This is what inspired our construction.

Remark 2.2. An object of interest in conformal geometry and, in particular, LCK geometry is the weight bundle. It is the real line bundle $L \longrightarrow M$ associated to the representation $\operatorname{GL}(2 n, \mathbb{R}) \ni A \mapsto|\operatorname{det} A|^{\frac{1}{n}}$ (see [6]). Then $L$ can be complexified and endowed with the Chern connection $\nabla_{0}+\sqrt{-1} \theta^{c}$ (where $\nabla_{0}$ is the trivial connection). It can be verified that $\omega_{0}=\sqrt{-1} \nabla^{2}$, and hence $\omega_{0}$ can be seen as the curvature form of this Chern connection. When $t=1$ and $M_{K}$ is the corresponding LCK OT-manifold, this construction gives the weight bundle introduced in Definition 2.2.

Remark 2.3. For any OT-manifold $M$, in addition to the Chern connection $\nabla=$ $\nabla_{0}+\sqrt{-1} \theta^{c}$, the weight bundle $L$ also admits the connection $\nabla_{0}+\theta$, which is flat because $d \theta=0$. It is clear that the $(0,1)$-part of $\nabla$ coincides with the $(0,1)$-part of this flat connection.

The following claim is obvious from the explicit form of $\omega_{0}$ (Theorem 2.1).

Claim 2.2. In the assumptions of Theorem 2.1, let $\tilde{\Sigma}$ be the holomorphic foliation on the covering $\tilde{M}_{K}=\mathbb{H}^{s} \times \mathbb{C}^{t}$ generated by the vector fields $\frac{\partial}{\partial z_{s+1}}, \ldots, \frac{\partial}{\partial z_{s+t}}$. Then:

(i) The foliation $\tilde{\Sigma}$ is $\Gamma$-invariant, hence it is obtained as the pullback of a holomorphic foliation $\Sigma$ on $M_{K}=\tilde{M}_{K} / \Gamma$.

(ii) The foliation $\Sigma$ is the null-space of the form $\omega_{0}$ constructed above.

Claim 2.3. Let $[K: \mathbb{Q}]$ be a number field of degree $n=s+2$, with $s$ real embeddings and two complex embeddings, $M_{K}$ the corresponding LCK OT-manifold, and $\Sigma \subset$ 
$T M_{K}$ the holomorphic foliation defined in Claim 2.2. Consider a complex closed subvariety $Z \subset M_{K}$. Then $\Sigma$ is tangent to $Z$ at any point of $Z$ :

$$
\forall z \in Z,\left.\quad \Sigma\right|_{z} \subset T_{z} Z
$$

Proof. The form $\omega_{0}$ has $(n-1)$ positive eigenvalues, where $n=\operatorname{dim}_{\mathbb{C}} M_{K}$, and its zero eigenspace at $z$ is $\left.\Sigma\right|_{z}$. Unless $(2.1)$ holds at $z \in Z$, the restriction $\left.\omega_{0}\right|_{Z}$ has $m=\operatorname{dim} Z$ positive eigenvalues at $z$. Then $\int_{Z} \omega_{0}^{m}>0$. This is impossible, because $\omega_{0}$ is exact.

Corollary 2.1. In assumption of Claim 2.3, let $\Sigma_{z}$ be a leaf of $\Sigma$ passing through $z \in Z$. Then $\Sigma_{z} \subset Z$.

\section{Complex subvarieties in LCK OT-manifold}

Using Corollary 2.1, we can easily prove the main result of this paper.

Theorem 3.1. Let $[K: \mathbb{Q}]$ be a number field of degree $n=s+2$, with s real embeddings and two complex embeddings, and let $M_{K}$ be the corresponding OT-manifold. Then $M_{K}$ has no non-trivial complex subvarieties.

Proof. Theorem 3.1 follows from Corollary 2.1 and the following more general proposition.

Proposition 3.1. Let $[K: \mathbb{Q}]$ be a number field of degree $n=s+2 t, t>0$, with s real embeddings and $2 t$ complex embeddings, and let $M_{K}=\mathbb{H}^{s} \times \mathbb{C}^{t} / \Gamma$ be the associated (non-Kähler) OT-manifold. Let $\Sigma \subset T M_{K}$ be the foliation defined in Claim 2.2. Consider a leaf of $\Sigma$, and let $Z$ be its closure. Then

(i) The preimage $\pi^{-1}(Z)$ of $Z$ in $\tilde{M}_{K}=\mathbb{H}^{s} \times \mathbb{C}^{t}$ contains the set

$$
Z_{\alpha_{1}, \ldots, \alpha_{s}}:=\left\{\left(z_{1}, \ldots, z_{s}, z_{s+1}, \ldots, z_{s+t}\right) \mid \operatorname{im} z_{i}=\alpha_{i}\right\}
$$

for some positive numbers $\left(\alpha_{1}, \ldots, \alpha_{s}\right) \in \mathbb{R}^{s}$.

(ii) Any complex subvariety of $M_{K}$ containing $Z$ must coincide with $M_{K}$.

Proof. The implication (i) $\Rightarrow$ (ii) is clear, because any complex manifold containing $Z_{\alpha_{1}, \ldots, \alpha_{s}}$ must have the same dimension as $M_{K}$. The proof of (i) is a bit more elaborate.

Let $\mathcal{O}$ be the ring of integers in $K$. By construction, the group $\Gamma=\pi_{1}\left(M_{K}\right)$ is a cross-product of the additive group $\mathcal{O}^{+}$of $\mathcal{O}$ with a subgroup of the multiplicative group $\mathcal{O}^{*}$. Let $\tilde{\Sigma}$ be the pullback of the foliation $\Sigma$ to $\tilde{M}_{K}=\mathbb{H}^{s} \times \mathbb{C}^{t}$. A leaf of $\tilde{\Sigma}$ is given as

$$
T_{t_{1}, \ldots, t_{s}}:=\left\{\left(z_{1}, \ldots, z_{s}, z_{s+1}, \ldots, z_{s+t}\right) \mid z_{i}=t_{i}\right\}
$$

for some $\left(t_{1}, \ldots, t_{s}\right) \in \mathbb{H}^{s}$. Let $\tilde{Z}:=\pi^{-1}(Z)$ be the preimage of the corresponding closure of a leaf of $\Sigma$. Clearly, $\tilde{Z}$ is the closure of $\Gamma\left(T_{t_{1}, \ldots, t_{s}}\right)$. Therefore, to prove Proposition 3.1 (i) it is sufficient to show that the closure of $\Gamma\left(T_{t_{1}, \ldots, t_{s}}\right)$ contains $Z_{\alpha_{1}, \ldots, \alpha_{s}}$. In fact, even the smaller group $\mathcal{O}^{+} \subset \Gamma$ will suffice, as seen from the following lemma, which proves Proposition 3.1. 
Lemma 3.1. Let $[K: \mathbb{Q}]$ be a number field of degree $n=s+2 t, t>0$ with $s$ real embeddings and $2 t$ complex embeddings, and $\tilde{M}_{K}:=\mathbb{H}^{s} \times \mathbb{C}^{t}$, equipped with the action of $\mathrm{O}^{+}$as in Subsection 1.2. Consider the subset

$$
T_{t_{1}, \ldots, t_{s}}:=\left\{\left(z_{1}, \ldots, z_{s}, z_{s+1}, \ldots, z_{s+t}\right) \mid z_{i}=t_{i}\right\}
$$

in $\tilde{M}_{K}$. Then the closure of $\mathcal{O}^{+}\left(T_{t_{1}, \ldots, t_{s}}\right)$ coincides with

$$
Z_{\alpha_{1}, \ldots, \alpha_{s}}:=\left\{\left(z_{1}, \ldots, z_{s}, z_{s+1}, \ldots, z_{s+t}\right) \mid \operatorname{im} z_{i}=\alpha_{i},\right\}
$$

with $\alpha_{i}:=\operatorname{im} t_{i}$.

Proof. Equivalently, we may state that the closure of an orbit of the standard action of $\mathcal{O}^{+}$in $\mathbb{H}^{s}$ is the set $\left\{\left(z_{1}, \ldots, z_{s}, z_{s+1}, \ldots, z_{s+t}\right) \quad \mid \quad \operatorname{im} z_{i}=\alpha_{i}\right\}$. This in turn is equivalent to the following.

Lemma 3.2. ( $c f . \quad[5$, Claim following Lemma 2.4]) Let $[K: \mathbb{Q}]$ be a number field of degree $n=s+2 t, t>0$ with $s$ real embeddings $\sigma_{1}, \ldots, \sigma_{s}$ and $2 t$ complex embeddings. Consider the additive group $\mathcal{O}^{+}$of the corresponding ring of integers. Let $\sigma: \mathcal{O}^{+} \longrightarrow \mathbb{R}^{s} \operatorname{map} \xi$ to $\sigma_{1}(\xi), \ldots, \sigma_{s}(\xi)$. Then the image of $\mathcal{O}^{+}$is dense in $\mathbb{R}^{s}$.

Proof. ${ }^{1}$ Let $K$ be a number field, $\mathcal{O}_{K}$ its ring of integers, $\mathfrak{P}$ the set of all prime ideals of $\mathcal{O}_{K}, V$ the product of all archimedean completions of $K$, and $V_{1}$ the product of some, but not all, archimedean completions. Denote by $\mathcal{O}_{\nu}$ the completion of $\mathcal{O}_{K}$ at $\nu \in \mathfrak{P}$, and let $K_{\nu}$ be the corresponding local field. Consider the adele space $\mathfrak{A}$, obtained as a subset of the product $V \times \prod_{\nu \in \mathfrak{P}} K_{\nu}$, where all components, except finitely many, belong to $\mathcal{O}_{\nu}$, and let $\mathfrak{A}_{1}$ be the image of projection of $\mathfrak{A}$ to $V_{1} \times$ $\prod_{\nu \in \mathfrak{P}} K_{\nu}$. Denote by $\tau: K \longrightarrow \mathfrak{A}_{1}$ the natural homomorphism, which is tautological componentwise.

From the Strong Approximation theorem (see [3] or [4, Theorem 20.4.4 $]^{2}$ ) it follows that the image $\tau(K)$ of $K$ is dense in $\mathfrak{A}_{1}$. Let

$$
\mathcal{O}_{\mathfrak{A}_{1}}:=\mathfrak{A}_{1} \cap\left(V_{1} \times \prod_{\nu \in \mathfrak{P}} \mathcal{O}_{\nu}\right)
$$

be the set of points of $\mathfrak{A}$, corresponding to the integer adeles. Clearly, $\mathcal{O}_{\mathfrak{A}_{1}}$ is open in $\mathfrak{A}_{1}$. Therefore, the intersection $\tau(K) \cap \mathcal{O}_{\mathfrak{A}_{1}}$ is dense in $\mathcal{O}_{\mathfrak{A}_{1}}$. On the other hand, $\tau(K) \cap \mathcal{O}_{\mathfrak{A}_{1}}$ consists of those elements of the number field which are integer at all non-archimedean places. This gives $\tau(K) \cap \mathcal{O}_{\mathfrak{A}_{1}}=\tau\left(\mathcal{O}_{K}\right)$. Therefore, the image of $\mathcal{O}_{K}$ to $V_{1}$ is dense.

Remark 3.1. The above argument actually proves that the image of $\mathcal{O}_{K}$ in the product $V_{1}$ of all archimedean completions of $K$ except one is dense in $V_{1}$.

\footnotetext{
${ }^{1}$ We are grateful to Marat Rovinsky, who kindly explained to us this proof.

${ }^{2} \mathrm{http}: / /$ modular.fas.harvard.edu/papers/ant/html/node $84 . \mathrm{html}$
} 


\section{Acknowledgments}

We are grateful to Katia Amerik for her support. Much gratitude to Marat Rovinsky for his invaluable help in proving the approximation lemma. Part of this work was done in Oberwolfach during the Research in Pairs programme; we are grateful to Oberwolfach Foundation for making it possible. Many thanks to Victor Vuletescu and Matei Toma for insightful email correspondence.

Liviu Ornea was partially supported by a PN2-IDEI grant, nr. 525, and by Tokyo Institute of Technology during summer of 2010.

Misha Verbitsky was partially supported by the RFBR grant 10-01-93113-NCNILa, RFBR grant 09-01-00242-a, Science Foundation of the SU-HSE award No. 10-090015 and AG Laboratory HSE, RF government grant, ag. 11.G34.31.0023.

\section{References}

[1] S. Dragomir and L. Ornea, Locally conformal Kähler geometry, in 'Progress in Math.' 155, Birkhäuser, Boston, Basel, 1998.

[2] M. Inoue, On surfaces of class $\mathrm{VII}_{0}$, Invent. Math. 24 (1974), 269-310.

[3] M. Kneser, Strong approximation, in 'Algebraic groups and discontinuous subgroups' (eds. A. Borel and G.D. Mostow), (Proc. Sympos. Pure Math., Boulder, Colo., 1965), Amer. Math. Soc., Providence, RI, 1966, 187-196.

[4] W. Stein, A brief introduction to classical and adelic algebraic number theory, 2004, electronic publication found at http://modular.fas.harvard.edu/papers/ant/html/ant.html

[5] K. Oeljeklaus and M. Toma, Non-Kähler compact complex manifolds associated to number fields, Ann. Inst. Fourier 55 (2005), 1291-1300.

[6] L. Ornea and M. Verbitsky, A report on locally conformally Kähler manifolds, Harmonic Maps and Differential Geometry, Contemp. Math. 542 (2011), 135-150. arXiv:1002.3473.

[7] M. Parton and V. Vuletescu, Examples of non-trivial rank in locally conformal Kähler geometry, Math. Z. (2010), DOI 10.1007/s00209-010-0791-5, arXiv:1001.4891.

[8] F. Tricerri, Some examples of locally conformal Kähler manifolds, Rend. Sem. Mat. Univ. Politec. Torino 40 (1982), 81-92.

[9] I. Vaisman, On locally and globally conformal Kähler manifolds, Trans. Amer. Math. Soc. 262 (1980), 533-542.

[10] M. Verbitsky, Theorems on the vanishing of cohomology for locally conformally hyper-Kähler manifolds, Proc. Steklov Inst. Math. 246 (2004), 54-78. arXiv:math/0302219.

[11] M. Verbitsky, Coherent sheaves on generic compact tori, math.AG/0310329, CRM Proc. Lecture Notes, 38 (2004), 229-249.

[12] M. Verbitsky, Stable bundles on positive principal elliptic fibrations, 17 pp., math.AG/0403430, also in Math. Res. Lett. 12(2-3) (2005), 251-264.

[13] V. Vuletescu, Sur l'existence de fibrés vectoriels stables sur les surfaces non-kählériennes, C. R. Acad. Sci. Paris Sér. I Math. 321 (1995), 591-593.

University of Bucharest, Faculty of Mathematics, 14 Academiei str., 70109 Bucharest, Romania and, Institute of Mathematics 'Simion Stoilow' of the Romanian Academy, 21, Calea Grivitei Street 010702-Bucharest, Romania

E-mail address: Liviu.Ornea@imar.ro, lornea@gta.math.unibuc.ro

Laboratory of Algebraic Geometry, SU-HSE, 7 Vavilova Str. Moscow, 117312, Russia

E-mail address: verbit@maths.gla.ac.uk, verbit@mccme.ru 